\title{
Comentario al laudo de BITARTU, Servicio Vasco de Arbitraje Cooperativo - CSCE, núm. abrev 20/2018, de 14 de enero de 2019. Expulsión de asociación socia de cooperativa ${ }^{8 x}$
}

\author{
Ińigo NAGORE* \\ Socio de LEGALKIDE ABOGADOS SLP. Árbitro de BITARTU. \\ Profesor ac. agregado UPV/EHU. Área de Derecho de Empresa
}

\begin{abstract}
Resumen: La asociación AXYZ es expulsada de una cooperativa de la que es socia por dejar de recibir los servicios de contabilidad y de control, manteniendo solamente el servicio de informatización y porque en los trámites de ejecución provisional de sentencias de procedimientos judiciales instados por los señores A y B contra la COOPERATIVA, esta ha tenido conocimiento de la contratación de los referidos señores por parte de AXYZ para la prestación del servicio de controladores, desde el día 5 de junio de 2017, como trabajadores a jornada completa. Se entiende que dicha contratación entra en colisión directa con los servicios que la COOPERATIVA presta a los ganaderos, con los consiguientes perjuicios económicos para la COOPERATIVA.
\end{abstract}

\& Dado el cambio de Ley aplicable desde el laudo se indicarán los artículos de la nueva Ley 11/2019 de Cooperativas de Euskadi, que resultarían hoy de aplicación.

* Correspondencia a/Corresponding author: Iñigo Nagore. Legalkide Abogados SLP. C/Ercilla $18-1^{\circ}$ izda. E-48009 Bilbao in@nagore.eu - https://orcid.org/0000-0002-4556-1747

Cómo citar/How to cite: Nagore, Iñigo (2020). "Comentario al laudo de BITARTU, Servicio Vasco de Arbitraje Cooperativo CSCE, núm. abrev 20/2018 de 14 de enero de 2019. Expulsión de asociación socia de cooperativa», GIZAEKOA - Revista Vasca de Economía Social, 17, 247-258. (https://doi.org/10.1387/gizaekoa.22235).

Recibido: 18/04/2020; aceptado: 30/04/2020.

ISSN 1698-7446 - elSSN 2444-3107 / (c) 2020 UPV/EHU

(c) (i) Esta obra está bajo una licencia

Creative Commons Atribución 4.0 Internacional 


\section{Relato del caso}

Se recibe en BITARTU, Servicio Vasco de Arbitraje Cooperativo (en adelante BITARTU), solicitud de arbitraje presentada el 5 de noviembre de 2018, formulada por AXYZ contra COOPERATIVA. Se comunica a las partes interesadas la Resolución de 7 de noviembre de 2018 de BITARTU, por la que, entre otras: se acepta de la tramitación de la solicitud de arbitraje, se acuerda su tramitación como abreviado al apreciar urgencia, se establece que el mismo debe ser resuelto en derecho y se designa árbitro en el procedimiento.

El árbitro acepta el nombramiento, el 22 de noviembre de 2018, notificándolo el mismo día a BITARTU y a las partes mediante acuerdo también del día 22 de noviembre, remitido el mismo día, el lugar de desarrollo de las actuaciones arbitrales y a AXYZ, en atención a que se había acordado su tramitación como abreviado por el presidente de BITARTU por decisión no rogada, a que formulara solicitud de arbitraje o se ratificara en la contenida en el escrito inicial. Igualmente se requiere a las partes que aportasen, si les era posible, una cuenta de email y/o un número de fax en que se le pudiera notificar, a fin de agilizar las notificaciones en el procedimiento.

El 3 de diciembre de 2018 se recibió correo electrónico de AXYZ, ratificando su anterior escrito y convirtiéndolo en escrito de demanda. En el citado escrito, se fija la pretensión del arbitraje en: «se dicte una resolución declarando la nulidad de la expulsión».

Las alegaciones en la que basa su pretensión pueden resumirse en: que AXYZ era socio de la demandada hasta la expulsión que se impugna, adoptada en Asamblea extraordinaria celebrada el 28 de julio de 2018 y notificada el 7 de septiembre de 2018, que existe sometimiento a BITARTU en base al artículo 61 de sus Estatutos sociales, que el expediente se inició con un pliego de cargos notificado el 28 de marzo al cual se remiten, que es nulo de pleno derecho por vulnerar el plazo estatutario de 60 días para faltas muy graves y el de prescripción de 90 días a contar desde que el Consejo rector tenga conocimiento de las faltas y que el Consejo rector tuvo conocimiento de la contratación de los controladores en el procedimiento de ejecución de sentencia y antes en una Asamblea informativa de la cooperativa, página cuatro del texto, el Consejo rector indica que tuvo conocimiento de los hechos en octubre de 2017, esto es, unos 150 días antes de notificarse el pliego de cargos. Reconoce que es cierto que han dejado de recibir los servicios de control de administración por parte de la cooperativa, pero niega los demás extremos. Señala que en mayo de 2017 en una solicitud de ayudas preparada por la Cooperativa para la Asociación, página 16, se indicaba que la Asociación contrataba de forma directa personal para la realización de los servicios de coordinación de control lechero, 
descargando en la cooperativa las labores de informatización de los datos y que, además, durante ocho meses, empleados de la Asociación han ido a la Cooperativa a entregar muestras y transmitir los datos, resultando difícil creer que nadie se hubiese enterado.

Alega que desde abril de 2017 la cooperativa dejó de prestar servicios a la Asociación, por otro tipo de cuestiones y estuvo a punto de dejarse sin sentido a la propia Asociación ya que la no realización de controles e inseminación controlada les hubiese abocado a la desaparición. Concluye indicando que el acuerdo de expulsión del Consejo rector de la cooperativa fue recurrido, desestimándose el citado recurso.

Igualmente, presentan cinco documentos: acuerdo adoptado por el Consejo rector de la cooperativa en sesión de 2 de mayo de 2018 sobre expediente sancionador, certificado relativo acuerdo adoptado en Asamblea General extraordinaria de la cooperativa celebrada el 24 de julio de 2018 sobre resolución de recurso contra el acuerdo de 2 de mayo, expediente sancionador de 22 de marzo de 2018, memorando de actuaciones en el conflicto con la empresa empleadora de los trabajadores contratados por la expulsada y otros temas a informar a los socios y copia del artículo 61 de los estatutos sociales relativo al arbitraje cooperativo. Por último, se indica dirección de correo electrónico y número de teléfono para facilitar las notificaciones en el procedimiento.

Por acuerdo del árbitro de 14 de diciembre de 2018, remitido el mismo día, se da por formulado el escrito de demanda y proposición de prueba (documental), y traslado de todo ello y sus documentos a la parte demandada, señalándose como fecha de la vista y práctica de la prueba en 9 de enero de 2019, a las 10 horas y, advirtiendo, que en la vista, las partes expondrían, por orden, lo que pretendieran y conviniera a su derecho, procediéndose a continuación a la práctica de las pruebas que considerándose oportunas y pertinentes así se acordasen, uniéndose al expediente los documentos y que una vez practicadas las mismas, el árbitro concedería la palabra a cada una de las partes para que, de manera verbal y concisa, expusieran sus conclusiones. Igualmente, se indicó a la Cooperativa que disponía de un plazo improrrogable de siete días naturales para señalar las personas que han de ser citadas por el árbitro a la vista, al objeto de que declaren en calidad de parte o testigo (artículo 62.1 del Reglamento de Bitartu). Igualmente se indicaba que podían reconvenir mediante escrito siempre que existiese conexión entre sus pretensiones y las que son objeto de la demanda principal, y ello del plazo de 15 días desde la recepción de la citación y, en todo caso, 10 días antes de la fecha prevista para la vista, no teniéndose por presentado en otro caso. También se volvió a requerir a la cooperativa para que facilitase una cuenta de correo electrónico y un número de teléfono y fax, a fin de agilizar las comunicaciones. 
El 17 de diciembre se remite correo electrónico en representación de la cooperativa, acompañando escritura de poder y señalando domicilio de notificaciones y datos de contacto y el 21 de diciembre se presenta escrito solicitando la citación por el árbitro de cinco testigos, así como planteando reconvención a la demanda formulada por la Asociación y acompañando a la misma tres facturas, solicitando en su reconvención que se condenase a la asociación al pago de la cantidad de $3.194,75 €$ correspondiente a las facturas por servicios prestados durante el año 2018.

El 28 de diciembre de 2018 el árbitro acuerda requerir al actuante en nombre de la Cooperativa que acreditase, antes de la vista del día nueve, su representación a efectos de arbitraje en alguna de las formas previstas en el Reglamento, al no estar comprendida dicha facultad en el poder presentado y a tener por planteada la reconvención con sus tres documentos anexos, sin perjuicio del requerimiento efectuado. No se pronuncia sobre la admisión, al no constar la representación, ni apreciarse, en ese momento procesal, conexión entre la citada reconvención y la demanda principal, posponiéndose la decisión a lo que se acordase durante la vista una vez oídas las partes. Respecto a la prueba solicitada por la Cooperativa, se admitieron cautelarmente los tres documentos presentados junto con la reconvención y los cinco testigos propuestos advirtiéndose que debían ser citados directamente por el proponente y que su testimonio se practicaría o no, en función de las cuestiones que se determinasen como objeto de prueba y de la relevancia de cada testigo con las mismas.

El 5 de enero de 2019 la Asociación demandante remite correo electrónico adjuntando contestación a la reconvención, y en el que se oponían a la admisión de la demanda reconvencional y proponían respecto de esta prueba de interrogatorio de parte en la persona del gerente de la cooperativa, y un testigo.

El 7 de enero el árbitro dicta acta de ordenación y prueba por la cual se tiene por presentado el escrito de contestación a reconvención y proposición de prueba sobre la misma, se admite el interrogatorio de parte así como un testigo, que se indica debe ser citado por el proponente y se advierte que en cualquier caso la prueba se practicará o no, a la vista de la admisibilidad de la reconvención y, en caso de admitirse, a la vista de las cuestiones que se determinasen como objeto de prueba y de la relevancia del testigo con las mismas.

El 9 de enero de 2019, en la sede de BITARTU-Servicio Vasco de Arbitraje Cooperativo, se practica la vista y práctica de prueba. Asisten la totalidad de las partes, esto es, la Asociación actora representada por su presidente y la Cooperativa representada por su Gerente y asistido por letrado. Con carácter previo, las partes acreditan sus representaciones, incorporán- 
dose copia de las mismas al procedimiento. Adicionalmente, se reconocen recíprocamente la representación. Se intenta la conciliación de las partes, sin éxito.

En primer lugar, se resuelve, tras audiencia de las partes, la admisibilidad o no de la reconvención planteada. Analizaremos el punto en la parte de Comentario. En segundo lugar, se fijan por el árbitro y las partes las cuestiones discutidas, una vez admitida por la Asociación que efectivamente en mayo de 2017 acordó que la cooperativa no llevase su contabilidad, no suministrándole los documentos necesarios para que la pudiese realizar y que de junio de 2017 se procedió a contratar personal propio para qué efectuase el servicio de control.

Por tanto, el debate se centraba en sí las presuntas faltas estaban prescritas y subsidiariamente, si la cooperativa estaba obligada a que la contabilidad y el servicio de control fuesen prestados por la cooperativa y el no hacerlo era supuesto de expulsión.

Por la Asociación se reitera la prueba documental aportada y la COOPERATIVA solicita presentar ocho grupos de documentos de los que son admitidos siete (se acompañó índice: actas de las sesiones del Consejo rector de la cooperativa de 9 de junio de 2017, 21 de febrero de 2018, 23 de marzo de 2018 y 2 de mayo de 2018 y de Asamblea General de 27 de junio de 2018, solicitud de la Asociación de la ayuda del Decreto Foral 52/2017, actas de la junta de gobierno de AXYZ de 29 de marzo de 2017 y Asamblea General extraordinaria de 8 de abril de 2017, diversos documentos del procedimiento laboral seguido por algunos socios de una sociedad TT que prestaba servicios a la COOPERATIVA y de la que algunos habían sido contratados por AXYZ, Convenio entre la COOPERATIVA y la Diputación Foral del año 2017 y Sentencia y documentación aportada en los juicios de despido interpuesto por un trabajador contra la COOPERATIVA que en el momento de la vista arbitral trabajaba en la Asociación).

Por parte del árbitro, se requiere en ese momento a las partes copia de los Estatutos sociales de la cooperativa, que son facilitados por esta y reconocidos por la demandante.

Las partes no impugnan los documentos aportados, aunque la Asociación manifiesta que algunos de los aportados de adversa, que a ella se referían, habían sido preparados por la Cooperativa y era la primera vez que los veía. Tras ello, se practica el interrogatorio de los testigos propuestos por la cooperativa. Las declaraciones fueron grabadas.

No existiendo más prueba pendiente de práctica se da a las partes la palabra para que formulasen sus conclusiones, lo cual efectúan. 


\section{Comentario}

\section{II.1. Requisitos de las reconvenciones}

En primer lugar, se resuelve en la propia vista, tras oír a las partes, sobre la admisibilidad o no de la reconvención planteada.

A la vista de que las cantidades reclamadas proceden de tres facturas que no han sido motivo de la expulsión, el árbitro resuelve denegando la admisión de la reconvención, por entender que ésta no cumple el requisito del artículo 61.1 del Reglamento del Consejo Superior de Cooperativas de Euskadi sobre procedimientos de resolución de conflictos las cooperativas vascas, por no existir conexión entre las pretensiones de la reconvención y las que eran objeto de la demanda principal (nulidad de la expulsión).

Debe considerarse adicionalmente que en un procedimiento abreviado la reconvención sólo debe caber sólo por cuestiones que a su vez puedan ser objeto de procedimiento abreviado y que en el mismo y por su especial procedimiento se puede reducir el derecho efectivo de defensa.

\section{II.2. Principio de congruencia y alcance del arbitraje}

El alcance del arbitraje queda limitado por el principio de congruencia, debiendo resolverse sólo lo que se plantea por las partes. Las pretensiones de la actora, están contenidas en su escrito de demanda: «se dicte una resolución declarando la nulidad de la expulsión».

El punto es relevante por cuanto que en el arbitraje afloran cuestiones controvertidas que no son objeto del procedimiento y que, aunque pueden ser hasta causa del mismo, no habían sido sometidas. La expulsión se producía a tenor de un pliego de cargos que contiene los motivos y fundamentación jurídica quedando las restantes cuestiones al margen.

Los hechos denunciados y que son objeto de la expulsión, constan en la documental aportada por la Asociación, en escrito de 22 de marzo de 2018.

«Hechos denunciados:

1. ${ }^{\circ}$ Que AXYZ ha dejado de recibir de la COOPERATIVA, los servicios de contabilidad y servicio de control, manteniendo solamente el servicio de informatización.

2. ${ }^{\circ}$ Que en los trámites de ejecución provisional de sentencias de procedimientos judiciales instados por los señores $A$ y $B$ contra la COOPERATIVA, ha tenido conocimiento de la contratación de los referidos señores por parte de $A X Y Z$ para la prestación del servicio de con- 
troladores desde el dia 5 de junio de 2017 como trabajadores a jornada completa. Dicha contratación entra en colisión directa con los servicios que la COOPERATIVA presta a los ganaderos vizcainos, con los consiguientes perjuicios económicos que está suponiendo a la COOPERATIVA.

(...)

Infracción cometida: los hechos denunciados pueden constituir unas faltas muy graves de las tipificadas en el artículo 20.1.a y 20.1. $f$ (de los estatutos sociales) por incumplimiento de las obligaciones señaladas en los artículos 12.c "participar en las actividades y usar los servicios que constituyen el objeto de la cooperativa» y en el artículo 12.d «no realizar actividades competitivas con el objeto social que desarrolla la cooperativa, ni colaborar con quienes las realice, ni contratar a empresas de la competencia de la cooperativa, salvo que estén expresamente autorizados por los administradores».

Sanción aplicable: dichos hechos pueden dar lugar a las siguientes sanciones: Por falta muy grave. A) Multa de 150,26 € (25.001 pesetas) a $360,61 €$ (60.000 pesetas) y suspensión de todos o algunos derechos sociales durante un plazo no superior a tres años e incapacitación para ostentar cargos del Consejo rector, hasta dos elecciones consecutivas. B) Expulsión."

Como se ha señalado el árbitro está vinculado por el pliego de cargos, sin que pueda entrar a valorar otros aspectos que a la vista de la prueba pudieran haber sido sancionados en plazos y formas diferentes. Igualmente, debe resolver conforme a la prueba practicada, aun cuando le quede la sensación de que la disputa entre las partes es más amplia y compleja.

\section{II.3. La prescripción de las faltas}

Se afirma por la Asociación de las faltas están prescritos por cuanto la Cooperativa conoce que las venía realizando desde hace más de 150 días al inicio del expediente sancionador. Por la Cooperativa, se niega este extremo manifestando que en cualquier caso se trata de una falta continuada y que a la fecha de la expulsión la Asociación sigue sin recibir los servicios de contabilidad y de servicio de control.

Pese a lo manifestado por la Cooperativa, consta en acta del Consejo rector de la misma, aportada por ella, de fecha 9 de junio de 2017, punto 7, «demandas de controladores de Z SC»: «El Gerente comenta como $Z$ no solo ha facturado a la COOPERATIVA en estos años sino a otros socios como AXYZ (...) Según esto comenta que el servicio vacuno de carne lo realiza desde el mes de mayo una persona que se ha contratado 
y en ovino, AXYZ decidió en Asamblea (8 de abril) hacer parada en el control lechero, ha asumido a tres de los cuatro trabajadores de $\mathrm{Z}$ y desde el 1 de junio ha vuelto a hacer controles e inseminaciones y ha rescindido el servicio de coordinación de la COOPERATIVA para que lo haga un controlador desde AXYZ. (...) A este respecto el señor $S$ comenta que AXYZ no se va a salir de la COOPERATIVA y, ante la demanda para que clarifique su decisión con respecto al servicio contable, el seńor $S$ certifica que en 2017 la COOPERATIVA no le va llevar la contabilidad».

Esto es, que desde al menos el 9 de junio de 2017, el gerente de la cooperativa y todo su Consejo rector conocen cuál era la postura de AXYZ respecto al servicio de control y a la contabilidad.

No obstante, entendiendo, que de ser obligatoria la recepción de dichos servicios, se siguen sin recibir a la fecha de apertura de expediente, y que la Asociación tampoco tiene ningún interés en que la situación cambiase (audiencia de la Vista), no se puede estimar la prescripción de los hechos objeto de sanción, ni del expediente, por cuanto que los mismos se mantienen en el tiempo, no tratándose de una falta puntual que pudiera estar prescrita.

Se reduce por tanto el debate a sí que los hechos denunciados, esto es la percepción del servicio de contabilidad y servicio de control, es de obligado cumplimiento para la cooperativa y su negativa a recibirlos puede ser objeto de expulsión.

\section{II.4. Análisis de la tipicidad de los hechos objeto de expulsión}

El artículo uno de los Estatutos sociales de la cooperativa la define como una "cooperativa del campo" y su artículo dos fija el objeto social en «la prestación de servicios técnicos y económicos a los socios y al sector agroalimentario». Tradicionalmente se denominaba como cooperativas del campo a las hoy llamadas cooperativas agrarias. La sociedad ha sido calificada como agraria por el Registro de Cooperativas de Euskadi.

El artículo 109 de la Ley de Cooperativas de Euskadi regula el objeto y actividades de las cooperativas agrarias estableciendo en el segundo párrafo de su punto primero:

"Los estatutos de la cooperativa podrán exigir, como requisito para adquirir y conservar la condición de socio, un compromiso de actividad exclusiva correspondiente al objeto social de aquellos."

(Hoy el artículo 112.1, in fine de la Ley 11/2019, de Cooperativas de Euskadi, "Los estatutos de la cooperativa podrán exigir, como requisito para adquirir y conservar la condición de persona socia, un compromiso de actividad exclusiva correspondiente a su objeto social.») 
Tal compromiso no es el motivo de la expulsión, ni se alega tan siquiera que conste en estatutos, lo cual es entendible a la vista de la gran extensión del objeto social, que viene a abarcar prácticamente cualquier actividad de sus socios.

Debe recordarse, a modo ilustrativo, que cuando el artículo 128 de la Ley de Cooperativas de Euskadi, regula las cooperativas de segundo o ulterior grado, exige que los Estatutos «deben incluir la enumeración de las facultades esenciales que, por ser precisas para el desarrollo de aquel objeto quedan transferidas a los órganos de dicha cooperativa».

(Hoy el artículo 146.1, segundo de la Ley 11/2019, de Cooperativas de Euskadi, "Los estatutos deberán incluir la enumeración de las facultades esenciales que, por ser precisas para el desarrollo de aquel objeto, quedan transferidas a los órganos de dicha cooperativa».)

Mediante el interrogatorio de la señora $T$ se acredita que los servicios se prestan a los socios a demanda de éstos, facturándoseles las horas recibidas, no existiendo un paquete de servicios predefinido, que la actora deja de enviar datos en junio de 2017, siendo la última declaración trimestral de IVA la correspondiente al primer trimestre de 2017 y que existe al menos otro socio de la cooperativa (JJ) que realiza de forma directa el servicio de control, aunque ya lo realizaba cuando se incorporó como socio a la cooperativa.

Consta igualmente en el propio pliego de cargos: «manteniendo solamente el servicio de informatización", hecho también recogido en la documental aportada por la cooperativa, acta de la Junta rectora del órgano, celebrada el 22 de marzo de 2018, punto cuarto, in fine, «en relación a la prestación de servicios se decide consultar a DFB sobre nuestra obligatoriedad de los servicios informáticos a AXYZ y que nuestro deseo sería no darle a no ser que nos obligue a ello». Esto es, consta que la Asociación seguía usando al menos los servicios informáticos prestados por la cooperativa, esto es realizando actividad cooperativizada.

Adicionalmente, la Asociación manifiesta que a partir de junio de 2017 la contabilidad la pasa a llevar de forma interna y que igualmente el servicio de control se internaliza, prestándose con personal propio.

Sobre que la contabilidad no ha sido llevada por empresas externa, no se intenta por parte de la Cooperativa prueba alguna, debiendo corresponder a la misma la carga de la prueba en caso de no aceptar el hecho alegado, máxime cuando del expediente de expulsión no se deduce lo contrario. La expulsión obedece, simplemente, a que ya no se realiza a través de la cooperativa.

Sobre la prestación del servicio de control directamente por la Asociación no existen dudas, por cuanto expresamente queda recogido en el 
expediente sancionador, así acuerdo adoptado por el Consejo rector de la cooperativa, en sesión de fecha 2 de mayo de 2018, sobre expediente sancionador (prueba documental aportada por la cooperativa).

Los anteriores hechos son tipificados en el pliego de cargos como faltas muy graves de los artículos 20.1 letras a y f, de los Estatutos sociales, esto es:

a) las operaciones de competencia, la colaboración o compra de bienes y servicios a empresas de la competencia, el fraude en las aportaciones al capital, la ocultación de datos relevantes, respecto a los útiles o herramientas, a la cualificación profesional del socio, o al proceso productivo en su conjunto (...)

f) el incumplimiento grave y reiterado de las obligaciones señaladas en los presentes estatutos, los acuerdos válidamente adoptados por los órganos de la cooperativa.

Poniendo la Cooperativa esta última letra en relación con lo dispuesto en el artículo 12 letras c "participar en las actividades y usar los servicios que constituye el objeto de la cooperativa» y $d$ «no realizar actividades competitivas con objeto social que desarrolla la cooperativa, ni colaborar con quien las realice, ni contratar empresas de la competencia de la cooperativa, salvo que estén expresamente autorizados por los administradores".

El laudo empezando por el primero de los hechos objeto de sanción, señala que llevándose internamente la contabilidad no se ve el encuadre en ninguno de ellos. La Asociación lleva su propia contabilidad, no ofrece servicios de contabilidad a terceros y no existe compromiso estatutario de actividad exclusiva con el objeto social de la Cooperativa, tal y como antes se ha desarrollado. No constando compromiso específico en Estatutos, ni acuerdo de la cooperativa válidamente adoptado, ni tan siquiera compromiso contractual, entiende que no parece que pueda ser objeto de sanción la decisión de llevar directamente su contabilidad. Por ello, se desestima el primer motivo de expulsión.

En cuanto al segundo de los hechos objeto de sanción, tenemos, adicionalmente: que la COOPERATIVA prestaba servicio a través de TT (indirectamente), sociedad que según el Gerente de la cooperativa (documental de la cooperativa, acta de su Consejo rector de 9 de junio de 2017, punto séptimo) había facturado en los últimos 20 años a otros clientes como la propia AXYZ; que, según queda acreditado en la sentencia ZZ/2018 del Juzgado de lo Social de refuerzo número cinco de Bilbao, procedimiento ordinario ZZZ/2017 (aportada por la cooperativa), TT tenía su domicilio social en $A X Y Z$ (hecho probado cuarto); que se presentaba a un concurso público anualmente convocado por la COOPERATIVA al 
que durante 20 ańos sólo ella se presentaba (hecho probado quinto) y que en el ańo 2017 no se presentó por lo que, el 28 de marzo de dicho año, la COOPERATIVA rescindió el contrato (hechos probados séptimo y octavo). Se prescinde por innecesarios a los efectos arbitrales de la incorporación de los restantes hechos probados de la sentencia.

La solución a la segunda cuestión es la misma que la recibida por la primera. Llevándose internamente el control no se ve el encuadre en ninguno de los tipos. La Asociación lleva su propio control, no ofrece servicios de control a terceros y no existe compromiso de actividad exclusiva de la asociación correspondiente al objeto social de la Cooperativa, tal y como hemos antes desarrollado. No constando compromiso específico en estatutos, ni en acuerdo de la cooperativa válidamente adoptado, ni tan siquiera compromiso contractual, no parece que pueda ser objeto de sanción la decisión de llevar directamente el control por cuanto que no queda acreditada la obligación de que lo tenga que llevar necesariamente la cooperativa. Por ello, se desestima el segundo de los motivos de expulsión.

Resuelto lo anterior, se hace constar, adicionalmente, en el laudo la vinculación que presentaba la Asociación con los trabajadores, constando en la documental aportada por la Cooperativa (vida laboral y la propia sentencia) que al menos dos trabajadores de los tres habían prestado servicios con anterioridad para ella, constando igualmente que la Cooperativa demandada había alegado la excepción de litis consorcio pasivo necesario respecto a la propia Asociación en la demanda presentada por los trabajadores en el Juzgado de lo Social. Esto es, que según la Cooperativa los trabajadores que la demandaban por laboralidad debían demandar también a la Asociación (fundamento de derecho segundo), excepción que fue desestimada (fallo de la sentencia). Planteada la excepción por la cooperativa, esta considera posteriormente que la contratación laboral de dichos trabajadores por la Asociación es causa de expulsión. Si la Cooperativa entendía que de existir relación laboral con los trabajadores demandantes esta debía ser con AXYZ, no tiene sentido expulsar a esta por formalizar finalmente dicha relación.

\section{II.5. Otras cuestiones no contenidas en el laudo}

El laudo no aborda la cuestión relativa a si el tipo de cooperativa elegido era el más adecuado para desarrollar la actividad pretendida por los socios. Debe remarcarse que a diferencia de las sociedades de capitales donde elegido el tipo (anónima, limitada...) será el objeto social el que marque la actividad sin mayores subtipos y condicionantes a los marcados con carácter general por su ley reguladora y los estatutos sociales en la ma- 
teria disponible, en el sistema cooperativo se distinguen clases de cooperativas estableciéndose un marco legal obligatorio y en ocasiones una parte dispositiva. Opciones que no usadas estatutariamente provocan el efecto interpretativo contrario.

La cooperativa se declaraba agraria y como tal estaba calificada. No obstante, en función de la actividad que se pretendía desarrollar, los socios que la componían y los compromisos que se decía querían imponer debería haberse valorado si la clase agraria presentaba ventajas sobre otras posibilidades y sobre todo recogerse, según procediera, en estatutos, reglamento o acuerdos de socios la totalidad de los compromisos que se pretendiesen exigirse los socios.

\section{Parte resolutiva del laudo}

Razonado lo anterior se estima la pretensión, declarándose la nulidad del acuerdo de expulsión de AXYZ, ratificado por la Asamblea General de la COOPERATIVA el 20 de junio de 2018.

En cuanto a las costas, se entiende que no existía mala fe o temeridad en ninguna de las partes por que se condena al pago por mitades, indicándose que sólo comprenden las que resultasen de las notificaciones, así como las que, en su caso, posteriormente se puedan derivar. 